\title{
El color en El Mundo cartesiano
}

\author{
Isabel Gamboa Cervantes \\ Departamento de Humanidades \\ Universidad Panamericana, Ciudad de México \\ mgamboa@up.edu.mx
}

[...] a fin de que la extensión de este discurso les sea menos aburrida, quiero engalanar una parte con la invención de una fábula, a través de la cual espero que la verdad no dejará de mostrarse suficientemente, y que no será menos agradable que si la expongo completamente desnuda (AT XI, 31; El mundo o Tratado de la luz, 75) ${ }^{1}$.

\section{I. Óptica en el sistema cartesiano}

Cuando Descartes publicó su famoso Discurso del método tenía 40 años y todavía recordaba, de la educación recibida en la Flèche, el encanto de las fábulas y la elevación de las memorables hazañas que despiertan el espíritu y logran que el juicio se forme (ver AT, VI, 4; Discurso del Método, 5).

La fábula que Descartes nos propone en El Mundo o Tratado de la Luz es -en palabras del filósofo- una hipótesis que pretende explicar cómo Dios pudo haber construido el

\footnotetext{
${ }^{1}$ Las obras de Descartes serán citadas según la edición canónica de Adam Charles y Paul Tannery (AT, volumen, página) y la traducción utilizada en castellano con la página.
} 
universo $^{2}$. La hipótesis comprende la explicación de todos los fenómenos del mundo físico: desde la formación de los astros, las mareas, los cometas y la naturaleza del fuego, hasta el funcionamiento del cuerpo humano y la explicación del conocimiento del mundo sensible ${ }^{3}$. Lo más notable es que las principales herramientas de su método son la geometría y el orden que los números heredan al mundo material.

El interés por ofrecer una explicación orgánica y unitaria, tanto del universo como del conocimiento humano, ha sido ejemplificado en el árbol de las ciencias que Descartes estampa en los Principios:

De este modo, la totalidad de la Filosofía se asemeja a un árbol, cuyas raíces son la Metafísica, el tronco es la Física y las ramas que brotan de este tronco son todas las otras ciencias que se reducen principalmente a tres: a saber, la Medicina, la Mecánica y la Moral [...] Y así como no se recogen los frutos del tronco ni de las raíces, sino sólo de las extremidades de las ramas, de igual modo la principal utilidad de la Filosofía depende de aquellas partes de la misma que sólo pueden desarrollarse en último lugar (AT, IX-II, 14; Carta prefacio de los Principios, 15; las negritas son mías).

Dicha intención se muestra tempranamente en El Mundo o Tratado de la Luz — redactado en Holanda en 1629, aunque su

\footnotetext{
${ }^{2}$ De hecho, al final del capítulo VII, Descartes comenta: «[...] no les prometo dar aquí demostraciones exactas de todas las cosas que diré; será suficiente con que les abra el camino, por el cual podrán encontrarlas por ustedes mismos, cuando se tomen la molestia de buscarlas. La mayor parte de los espíritus pierde el gusto cuando se le dan las cosas demasiado fáciles [...] Si bien me limitaré a proseguir la descripción que he comenzado como si no tuviera otra intención que la de contarles una fábula» (AT XI, 48; El Mundo o Tratado de la Luz, 93).

${ }^{3}$ También en la tercera parte de Los principios insiste en el carácter hipotético de la propuesta. El criterio de elección es claro: «podemos elegir aquella hipótesis que deseemos, siempre y cuando todo lo que sea deducido sea enteramente acorde a la experiencia» (AT IX-II, 99; Principios, 149).
} 
publicación se haya postergado por la condena de Galileoy en la Dióptrica, escrita en 1626. Como se verá más adelante, las leyes de la naturaleza y, en concreto la explicación de la luz y la percepción del color, son frutos de aquella segunda rama del árbol de la Filosofía, nutrido por la savia de los principios metafísicos. Por este motivo, ambas obras son una muestra en germen de la unidad explicativa pretendida por Mounsier Descartes.

El Mundo o Tratado de la Luz es considerado, no tanto como una obra de física, sino como un texto de filosofía de la naturaleza, pues en ella encontramos - considerando los tres enfoques que Benítez (ver 1986; 38) recomienda- la explicación del mundo natural a partir del modelo de la óptica mecanicista. En el estudio que precede a la traducción, Benítez afirma que la diversidad de tópicos abordados sobre filosofía de la naturaleza tiene como hilo conductor el paradigmático fenómeno de la luz. Paradigmático porque es el criterio para clasificar a los cuerpos del universo en tres géneros: sol y estrellas fijas; cielos; tierra y cometas ${ }^{4}$; y porque la luz y el calor serán considerados, no como cualidades objetivas de los cuerpos, sino como modos subjetivos de percibir el movimiento de las diversas partes de una misma materia homogénea. Como se verá más adelante, la notable influencia que el mecanicismo aportó en la comprensión de la materia y el movimiento fue un punto de inflexión en la trayectoria de la filosofía de la naturaleza moderna.

En la fábula sobre la génesis del mundo encontramos las leyes básicas del mecanicismo entremezcladas con la teoría cartesiana de la luz. De todas las explicaciones que se reco-

\footnotetext{
${ }^{4}$ Esta misma hipótesis será retomada en la tercera parte de Los principios para matizar algunos puntos.
} 
gen ahí, todavía existen dudas sobre la autoría, en concreto, de una de las más simples y fundamentales leyes de la óptica, a saber, la ley de la refracción. Sobre su originalidad hay diferentes objeciones ${ }^{5}$. Al margen de esta discusión, Shea (ver 1993; 213- 215) nos introduce en el contexto parisino de 1625 con el que Descartes se encontró: un ambiente inquieto por los temas de óptica, tras la publicación de Quaestiones in Genesim en 1623 y de La Verité de Sciences en 1625. En ambos textos del fraile Mersenne se resumía el status quaestionis de las tres ramas de la óptica entonces conocidas: la óptica, que estudiaba la propagación de la luz; la catóptrica, que estudiaba la reflexión de los espejos; y la dióptrica, que estudiaba la refracción.

Si bien lo más probable es que Descartes no haya sido el primero en formular la ley de la refracción, se cree que él fue el primero en ofrecer una justificación completa y sistemática de esta ley ${ }^{6}$. Además, su prueba partía de una perspectiva totalmente diferente a las anteriores, pues su teoría de la radiación de ésta se centraba en la idea de que la luz consiste en los impulsos mecánicos transmitidos a través de medios

\footnotetext{
${ }^{5}$ Los anglófonos, por ejemplo, creen que Descartes la descubrió durante su estancia en París entre 1625 y 1628. Sin embargo, no dejó una explicación del procedimiento utilizado para hallarla. Por otro lado, el consenso de la mayoría se lo atribuye al físico holandés Willebrord van Roijen Snell (Leiden, 1580-1626) en el año 1621, aunque durante mucho tiempo se le adjudicó a Descartes, porque estaba incluida en el Discurso del método (1637). No obstante, en 1703 Huygens publicó las investigaciones de Snell junto con las suyas en su Dióptrica. Los estudios más recientes han mostrado que hubo otro personaje que también la descubrió: Ibn Sahl, matemático y físico de la corte de Bagdad del siglo X, quien la demostró en su tratado de óptica geométrica Kitab al-Harraqat (El libro sobre los instrumentos incendiarios), seis siglos antes que Snell (ver Cerantola 2004; 58).

${ }^{6}$ Incluso en lo referente a la refracción, fue atacado inmediatamente después de la publicación de la Dióptrica. En esto, Pierre de Fermat fue, sin lugar a dudas, el más efectivo y persistente, pues dudaba de la validez misma de la ley del seno y, al parecer, permaneció dudoso hasta 1662 cuando, irónicamente, demostró esta ley a su modo. También sospechaba del procedimiento seguido por Descartes, pues consideraba que había acomodado los recursos de la prueba para llegar a la conclusión (ver Smith 1987; 5-7).
} 
transparentes.

Si bien antes se pensaba que el color era una modificación de la materia, i.e. una cualidad metafísica del objeto susceptible de percibirse cuando la luz lo alumbra, la novedad de la explicación cartesiana radica en considerar al color como resultado del reflejo de la luz en un objeto, fenómeno relacionado con el 'giro' de las partículas de la luz.

En este sentido, las especulaciones del pensador de La Haya marcaron un decisivo avance en comparación con las explicaciones previas. Los pensadores que le precedieron se contentaban con explicar el color como el resultado de la mezcla de la luz y la obscuridad, o de determinada combinación de colores primarios en distintas proporciones. $\mathrm{Nu}$ merosos historiadores de la ciencia (ver Sabra 1981; 46-48) afirman que la teoría de la propagación instantánea de la luz era anterior. No obstante, lo notable es que Descartes fue el primero en sostener una teoría mecánica que explicaba el fenómeno a través de un medio material; recurso que se distinguía del uso de las 'formas' de Ibn al-Haytham y Witelo o las 'especies' de Grosseteste, Roger Bacon y Kepler. De hecho, las analogías mecánicas fueron utilizadas para explicar los fenómenos ópticos mucho antes de Descartes, pero él fue el pionero en explicar que la luz en sí misma no es más que una propiedad mecánica del objeto luminoso y de la transmisión en el medio (ver Straker 1970; 1074). En este sentido, se puede afirmar que la teoría de Descartes sobre la luz es un legítimo punto de inicio de la física óptica moderna.

A pesar de las objeciones planteadas a sus explicaciones, la influencia del caballero de la Turena en la historia de la óptica es innegable y ha sido reconocida por filósofos de la ciencia como Westfall (ver 1962; 347), quien señala que la investigación de Robert Boyle (Experiments and Considerations 
Touching Colours, 1664); la de Robert Hooke (Micrographía, 1665); y la decisiva incursión de Isaac Newton en la óptica experimental, son tres de los muchos ejemplos que manifiestan la importancia del mecanicismo para la teoría óptica.

\section{La luz y el color bajo la óptica mecanicista}

Descartes presenta en El Mundo o Tratado de la Luz la hipótesis sobre la racionalidad divina, proyectando una creación alevosamente sintonizada a nuestra capacidad para conocer los fenómenos de la naturaleza. La intención se muestra explícita en el capítulo 6, cuando invita a que la imaginación trascienda las barreras espacio-temporales y se sitúe en aquel inicio:

Permitid pues que vuestro pensamiento, durante un tiempo, salga fuera de este mundo para acceder a otro totalmente nuevo, que yo haré nacer en su presencia en los espacios imaginarios [...] Supongamos que Dios crea de nuevo alrededor nuestro tanta materia que, hacia cualquier lado a que nuestra imaginación pueda extenderse, no perciba ya ningún lugar que esté vacío [...] Ahora bien, puesto que nos tomamos la libertad de fingir esta materia según nuestra fantasía, atribuyámosle, si os parece, una naturaleza en la que no haya nada más que lo que cada uno pueda conocer tan perfectamente como sea posible (AT, XI, 32-33; El mundo o Tratado de la Luz, 77-79; las negritas son mías).

El supuesto básico del universo cartesiano es el mecanicismo, dado que la explicación se centra en describir la transferencia del movimiento relegando así la justificación causal al orden metafísico: Dios como causa última de todo cuanto existe, tanto materia como movimiento. 
La explicación que pretende Descartes es ordenada y sintética, pues parte de la unidad material del universo considerándola desde su faceta cuantitativa. Tal reducción permite explicar la materia en términos geométricos: con forma, tamaño, y susceptible de desplazamientos rectilíneos, los más simples que hay. En esta reducción se pretende simplificar la complejidad del mundo dejando de lado todo aspecto cualitativo. Para Mounsieur Descartes el mundo es un pleno de materia compuesto de pequeños corpúsculos en cuya estructura básica se distingue la figura, el tamaño, disposición y velocidad. Por tanto, la homogeneidad material que se deriva de la teoría corpuscularista no es de orden cualitativo, sino cuantitativo. Aunque persiste la dificultad para considerar un pleno constituido por partes, es importante señalar que en este pleno tridimensional no existe el vacío, es decir, no hay espacio sin materia.

El movimiento se explica porque los corpúsculos no dejan de moverse - en algunos cuerpos con más violencia y en otros con menor-dado que el pleno de materia existente en el universo transmite y recibe el movimiento según las diversas densidades y cohesiones de los elementos: fuego, aire y tierra. Cada uno distinto según sus características cuantitativas (ver AT, XI, 24-27; El Mundo o Tratado de la Luz, 69-74).

Junto con la materia, Dios creó el movimiento que se transmite entre los cuerpos desde el inicio, conservándose siempre en la misma cantidad: la materia no tiene movimiento en sí misma, por lo que su única capacidad reside en recibirlo y transmitirlo; ni lo genera, ni lo anula. De esto se sigue que el movimiento se realice únicamente por contacto. Al no existir espacios vacíos, todo movimiento implica choque. Cualquier tipo de causalidad a distancia o intrínseca queda anulada del esquema cartesiano. 
Esta comprensión geométrica de la materia facilita la explicación de la transmisión de la luz y la percepción del color, en la medida en que se entienden como dos fenómenos más, igualmente explicables a partir de tres leyes básicas expuestas en el mismo Tratado.

Primera ley del movimiento o ley de la inercia:

Toda parte de la materia, individualmente, continúa siempre existiendo en un mismo estado, mientras el encuentro con las otras no la obligue a cambiarlo; [...] si se ha detenido en algún lugar no saldrá jamás de él si las otras no la desplazan; y si ha comenzado en alguna ocasión a moverse, continuará haciéndolo con la misma fuerza hasta que las otras la detengan o la retrasen (AT, XI, 38; El Mundo o Tratado de la Luz, 84).

Segunda ley del movimiento o ley de la resistencia:

Cuando un cuerpo empuja a otro, no podría darle ningún movimiento si no perdiera al mismo tiempo proporcionalmente el suyo, ni quitárselo sin que el suyo aumente otro tanto (AT, XI, 41; El Mundo o Tratado de la Luz, 86).

Tercera ley o ley del movimiento rectilíneo:

[...] mientras que un cuerpo se mueve, aunque su movimiento se dé a menudo en línea curva, y que no pueda jamás hacer ninguno que no sea en alguna forma circular, [...] de cualquier modo cada una de sus partes individualmente, tiende siempre a continuar el suyo en línea recta (AT, XI, 44; El Mundo o Tratado de la Luz, 89).

Tal como se dibuja en el árbol de la Filosofía expuesto en los Principios, las leyes físicas y sus frutos mecánicos tienen su fun- 
damento arraigado en la metafísica cartesiana. El garante de la inmutabilidad de las leyes, la regularidad de los fenómenos, la persistencia del movimiento rectilíneo, así como de la capacidad por conocer el mundo sin miedo al engaño, es Dios ${ }^{7}$.

Las tres leyes resultan importantes para explicar la transmisión de la luz; pero de la tercera se deriva que todo movimiento se transmite por contacto y de manera instantánea. En este sentido, se afirma que «la doctrina de la propagación instantánea es una consecuencia necesaria de la concepción de Descartes, en la cual el medio sirve como vehículo de la luz. La naturaleza de dicho medio está determinada en sí misma por la definición de materia cartesiana» (Sabra 1981; 55).

Aunque esta visión plenista no se libra de serias dificultades para explicar el movimiento, es un hecho que Descartes legó considerables aportaciones respecto a la explicación física del movimiento negando, por ejemplo, las causas que no fueran de orden mecánico y la teoría de los lugares naturales.

La explicación de la transmisión de la luz y la percepción del color están íntimamente relacionadas con las leyes de la naturaleza; tanto que, para explicar cómo los rayos de luz penetran en los ojos y cómo son desviados por diversos cuerpos, Descartes propone tres comparaciones:

Sin duda alguna, habéis visto la necesidad de utilizar un bastón para guiaros cuando caminabais sin luz por lugares difíciles durante la noche [...] Para establecer una comparación a partir de esto, deseo que penséis que la luz no es otra cosa en los cuerpos,

\footnotetext{
${ }^{7}$ «Así, estas dos reglas se siguen manifiestamente, de esto sólo, que Dios es inmutable, y que al actuar siempre del mismo modo produce siempre el mismo efecto. Pues suponiendo que ha puesto cierta cantidad de movimiento en toda la materia en general desde el primer momento en que la creó, es necesario reconocer que la conserva siempre igual, o no creer que actúa siempre de la misma manera» (AT. XI, 43; El Mundo o Tratado de la Luz, 88).
} 
que son llamados luminosos, que un cierto movimiento o una acción muy rápida y muy viva que se dirige hacia nuestros ojos a través del aire y de los otros cuerpos transparentes, de igual forma que el movimiento o la resistencia llega a su mano a través del bastón (AT, VI, 84; Dióptrica, 61; las negritas son mías).

Esta primera comparación con el bastón pretende resaltar la transmisión mecánica de la luz y no tanto que el movimiento sea instantáneo; a la vez, sugiere que nuestra percepción nos da un acceso directo a los objetos. El ciego siente el objeto en sí mismo y no una imagen o copia de éste. Dicho en otros términos, sentimos los objetos por medio de los rayos de luz que tocan y hacen presión a nuestros ojos. Como consecuencia de este movimiento, los colores son «las diversas formas en que los mismos [cuerpos] reciben y reflejan la luz contra nuestros ojos» (AT, VI, 85; Dióptrica, 61). Aunque esta primera analogía se muestra burda y simple, resulta válida para quien considera que las causas mecánicas son la única explicación del movimiento natural y de los fenómenos que percibimos. La reducción de aspectos cualitativos, como el color, se concreta en otro susceptible de cálculos de orden geométrico-matemáticos:

[...] si consideráis que las diferencias constatadas por un ciego entre diversos árboles, piedras, agua y cosas semejantes por medio de su bastón no parecen menores de lo que son para nosotros aquellas que existen entre el rojo, el amarillo, el verde y todos los otros colores. Y sin embargo, todas aquellas diferencias no son otra cosa en todos estos cuerpos, que las diversas formas de mover o de resistir a los movimientos de este bastón (AT, VI, 85; Dióptrica, 62). 
La última deducción que Mounsieur Descartes consigue de la analogía del ciego y el bastón, es la necesidad de desechar aquella teoría de las 'especies intencionales', ya que «no es necesario suponer que fluya cosa alguna material desde los objetos hasta nuestros ojos para hacernos ver los colores y la luz, ni que haya nada en estos objetos que sea semejante a las ideas ni sentimientos que de ellos tenemos» (AT, VI, 85; Dióptrica, 62). El movimiento como causa próxima única es suficiente para dar cuenta de los fenómenos físicos. En este caso, la resistencia nada tiene de semejante con las ideas que concebimos.

La segunda analogía que pretende explicar la naturaleza de la luz se abre paso a partir de una concesión evidente: «puesto que hay una gran diferencia entre el bastón de este ciego y el aire o los otros cuerpos transparentes, a través de los cuales vemos, es preciso que recurra a establecer otra comparación» (AT, VI, 86; Dióptrica, 62). A partir de la comparación de un barril de vino en fermentación expone, en paralelo a la tercera ley de la naturaleza, cómo se da el movimiento rectilíneo de la luz ${ }^{8}$. Esta analogía, sin embargo, no resulta relevante para la explicación del color, pues se enfoca en la descripción del movimiento multidireccional de la luz utilizando la comparación del zumo de las uvas, que tiene la propiedad líquida y expansiva de la que carece el bastón.

En tercer lugar, el modelo del juego de pelota intenta explicar los movimientos de reflexión y refracción de la luz.

\footnotetext{
${ }^{8}$ En la tercera parte de los Principios, ofrece un ejemplo similar, i.e., el del recipiente con bolas de plomo pero considerando el fenómeno luminoso como un efecto del medio cuyo movimiento se transmite gracias a la naturaleza líquida de los cielos: «[...] es preciso señalar que la fuerza de la luz, en razón de cuya explicación escribo todo esto, no consiste en la duración de algún movimiento, sino sólo en que estas pequeñas bolas son presionadas y tienden a moverse hacia algún punto» (AT, IX-II, 108; Principios, 165).
} 
La analogía nos pide que pensemos en una pelota golpeada por una raqueta con diferentes ángulos: en ocasiones recto y en otras dándole algún efecto para que rote. Estos efectos dependen de la composición de cada cuerpo, pues unos amortiguan, otros reflejan, otros reciben y otros reflejan confusamente los rayos de la luz. De este último caso, unos reflejan sus rayos sin cambio alguno - v.g. los llamados cuerpos blancos-y otros aportan un cambio «semejante al que recibe el movimiento de una pelota cuando se la golpea con efecto, a saber, los que son rojos, amarillos, azules o de otro color tal como éstos» (AT, VI, 92; Dióptrica, 66).

Con este ejemplo, se suma un movimiento más al rectilíneo, pues las partículas, además de chocar con cuerpos de diversas densidades y con superficies de diferentes formas, están provistas de un movimiento que las hace girar en torno suyo; es decir, mientras viajan se mueven alrededor de su centro y en el radio de su fuerza rotacional se determinan nuestras sensaciones del color. A partir de ambos movimientos, Descartes explica en el capítulo 8 de Los Meteoros que la naturaleza de los colores consiste en:

[...] que las partes de la materia sutil que transmiten la acción de la luz tienden a girar con más fuerza que a moverse en línea recta; de modo que las que tienden a girar con mucha mayor fuerza, dan lugar a la formación del rojo, y las que no tienden a girar sino un poco más fuerte, causan el amarillo. Por el contrario [...] el verde aparece donde no giran con mucha menos lentitud y el azul cuando giran con mucha menos lentitud. Ordinariamente, en los límites de este azul se encuentra el encarnado que, dándole vivacidad y brillo, lo cambia en violeta o color de púrpura (AT, VI, 328; Dióptrica, 251). 
La liquidez de los cielos es la condición que posibilita la transmisión de la luz, ya que las finas partículas de esta 'materia sutil' llenan todos los espacios y se acomodan fácilmente9 Aunque en la analogía con la pelota de tenis que comienza a girar en el momento que la raqueta la golpea haya varias disanalogías, queda claro que tal explicación del color implica el movimiento de las partículas y su reflexión en las superficies.

La explicación cartesiana del color refuerza la consideración de una propiedad física en los objetos como causa de que reflejen luz de un determinado modo, pues depende de la configuración y movimientos de las partículas que conforman al objeto ${ }^{10}$. En este sentido, Wolf señala que los objetos sí poseen un color dado que tienen en su superficie ciertas propiedades estructurales que dan cuenta de la refracción de la luz (ver 1993; 45-46). No obstante, Descartes se adelanta y afirma no tener todos los elementos y recursos necesarios para determinar «en qué consiste la naturaleza de cada uno de estos colores y hacerlo ver mediante la experiencia» (AT, VI, 92; Dióptrica, 66). Cuestión que tuvo que esperar a desarrollos científicos posteriores.

Sabra emite un juicio positivo sobre la explicación cartesiana de la luz, en tanto que explica que la luz en sí misma no es más que una propiedad mecánica del objeto luminoso y de la transmisión en el medio (ver 1981; 48). Además, loa

\footnotetext{
${ }_{9}^{9}$ La explicación completa de los principios del mundo visible se encuentra ampliamente desarrollada en la tercera parte de Los principios de la filosofía; sin embargo, no lo abordaremos aquí por razones de espacio. Basta señalar que su cosmovisión mecanicista no satisface del todo problemas que heredará a la óptica, tales como la explicación instantánea en un medio mecánico o la justificación experimental del espectro.

${ }^{10}$ Esto no excluye que su postura crítica ante la distinción hecha por algunos filósofos escolásticos entre el color 'real' de los cuerpos y el 'aparente' color del arco iris haga de este tópico de filosofía natural el estandarte paradigmático de la metafísica cartesiana (ver Westfall $1962 ; 340)$.
} 
la continuidad argumentativa que hay entre El mundo, donde establece los principios, la Dióptrica y las obras posteriores: en las dos primeras asume los principios y los procesos a partir del uso de las analogías que, sin embargo, son ciertamente poco rigurosas. Estos tres modelos explicativos de la luz cumplen una función precisa dentro del mundo cartesiano, pero padecen de las limitaciones propias de toda representación metafórica, además de carecer de una verificación ${ }^{11}$.

A pesar de las iniciales pretensiones para demostrar la refracción de la luz geométricamente y a priori, así como la transmisión de la luz a través del medio material en el universo, debemos a la confiada correspondencia con Marin Meresenne, el reconocimiento de los alcances explicativos de su sistema y su método. En ésta acepta haber demostrado los principios de la física y la metafísica, pero reconoce que tales demostraciones geométricas son cuestiones que pertenecen a la física, algo imposible para él (ver AT, II, 31; a Mersenne, del 1 de marzo de 1638. Y la respuesta en AT, II, 142; a Mersenne, del 27 de mayo de 1638) ${ }^{12}$.

El color, sin embargo, no se explica únicamente por la velocidad y la rotación de pequeñas partículas que se mueven en línea recta. El color es principalmente una sensación de orden epistemológico. En esta línea sabemos que la propues-

\footnotetext{
${ }^{11}$ De ahí que autores como Stansfield le recriminen moverse en diferentes niveles argumentativos, pues en el caso de la teoría de la refracción expuesta en la Dióptrica, es presentada de tal modo que se presta únicamente a la aceptación o al rechazo, pero no a la refutación (ver Stansfield 1984; 502).

${ }^{12}$ Incluso refuerza la respuesta a Mersenne señalando: «Que si únicamente las pruebas geométricas pueden ser llamadas demostraciones, entonces debe decirse que Arquímedes nunca demostró nada de la mecánica, o Witelo en la óptica, o Ptolomeo en la astronomía» (AT, II, 142; a Mersenne, del 27 de mayo de 1638). Como siempre, el argumento base del sistema cartesiano es apelar a la certeza que brindan aquellas verdades simples e intuitivas que son coherentes con el sistema y están libres de error lógico. Éstas serán el garante epistemológico de su método.
} 
ta epistémica cartesiana se asienta en una fisiología peculiar; la biología se considera como una rama más de la mecánica, ya que los organismos vivos y sus funciones son explicables en términos de la física y química de sus partes.

En El tratado del hombre ${ }^{13}$, Descartes introduce la hipótesis del hombre-máquina, proponiendo así un nuevo paradigma. En este campo se puede considerar a Descartes como un pionero, pues intentó explicar cómo se da la transmisión de la información visual incluyendo su conexión con el cerebro, las terminaciones nerviosas y la glándula pineal.

La visión de los colores ocurre cuando los rayos del sol se concentran en nuestras retinas causando movimiento en el nervio óptico que se transfieren al cerebro y a la glándula pineal, donde actúan sobre el alma, pues «sabemos que es el alma quien siente y no el cuerpo» (AT, VI, 109; Dióptrica, 81).

Pues si bien es verdad que el sentimiento que tenemos de la luz es causado por el movimiento o inclinación a moverse de alguna materia que toca nuestros ojos, [...] también es cierto que los diversos movimientos de esta materia deben producir en nosotros diversos sentimientos. Y como no puede darse otra diversidad en estos movimientos distinta de la expresada, tampoco localizamos otra por experiencia en nuestros sentimientos que la de los colores (AT, VI, 327; Dióptrica, 251).

Descartes dedica veintitrés artículos de la tercera parte de $E l$ tratado del hombre a explicar la estructura del ojo, la función

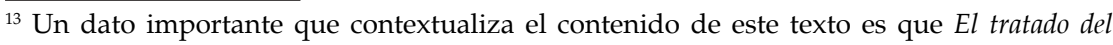
hombre — según el testimonio de Clerselier- era el capítulo XVIII de El Mundo o Tratado de la $L u z$ y no figuraba como una obra independiente entre los papeles recogidos por Chanut a la muerte de Descartes.
} 
de sus partes, el efecto que produce la forma de cada una de ellas y cómo captamos la figura, la distancia y el tamaño de los objetos. La razón de este interés está enunciada en el primer párrafo de la Dióptrica ${ }^{14}$ y refuerza aquella intención filantrópica enunciada en el árbol de la Filosofía de los Principios.

Todas las cualidades que percibimos en los objetos de la vista pueden ser reducidos a las seis principales siguientes: la luz, el color, la situación, la distancia, el tamaño y la figura. Primeramente y en relación con la luz y el color, que propiamente se relacionan de modo exclusivo con el sentido de la vista, es preciso pensar que nuestra alma es de tal naturaleza que es la fuerza de los movimientos, localizados en aquellos lugares del cerebro en donde se originan los pequeños filamentos de los nervios ópticos, la que causa el sentimiento de la luz, y que es el modo en que se producen estos movimientos el que causa el sentimiento del color (AT, VI, 131; Dióptrica, 97; las negritas son mías).

En su explicación describe el mecanismo de la sensación que experimentamos como dependiente del modo en que corren los movimientos de los nervios ópticos a través de los espíritus animales que confluyen en el cerebro. Como señala Wolf, esto depende a su vez, de ciertas variables físicas (i.e., mecánicas): la cantidad de luz en los objetos, su distancia, el tamaño de la pupila y la cantidad de espacio que los rayos de cada parte del objeto ocupa en la retina ${ }^{15}$. En virtud de dichas

\footnotetext{
14 «Toda la conducta de nuestra vida depende de nuestros sentidos. La vista es el más universal y el más noble de todos los sentidos y no existe duda alguna de que las invenciones que puedan contribuir a dilatar su poder han de ser las más útiles» (AT, VI, 81; Dióptrica, 59). ${ }_{15}$ «Así mismo la visión distinta de los colores no depende solamente de que todos los rayos, procedentes de cada punto del objeto, se reúnan poco más o menos en otros tantos puntos en el fondo del ojo, así como de que no se dirijan otros rayos a estos mismos puntos desde otras partes, tal como anteriormente ha sido indicado, sino que también depende de la mul-
} 
variables, se perciben los diferentes colores de un objeto a través de las terminaciones nerviosas que ocupan la porción de la retina en que esa imagen se $\operatorname{proyecta}^{16}(1993 ; 62)$.

La cuestión sobre la percepción del color tiene una dificultad extra si se considera el dualismo radical del pensador francés; principio que decide asumir en su sistema, pero sin resolverlo satisfactoriamente ${ }^{17}$. En esta línea, el problema de cómo la máquina del cuerpo puede ser excitada por los objetos exteriores debe analizase, por lo menos, en dos planos:

a) El fisiológico propio de la res extensa: basado en la disposición de pequeños filamentos que provienen de la parte más interna del cerebro y que pueden ser movidos fácilmente por los objetos que afectan a los sentidos. En este complejo mecanismo se abren ciertos poros del cerebro que permiten la acción de los espíritus animales dirigiéndose hacia los nervios y músculos para producir en la máquina: «movimientos que son en todo iguales a los que nosotros estamos naturalmente inclinados a realizar cuando nuestros sentidos son estimulados de igual forma» (AT, XI, 141; Tratado del hombre, 48).

b) El de la unión, propio del ser humano real: a diferencia de la res cogitans, al ser humano real —aquella alma puesta en la máqui-

\footnotetext{
titud de pequeños filamentos del nervio óptico que están en el espacio que ocupa la imagen en el fondo del ojo» (AT, VI, 134; Dióptrica, 99).

${ }_{16}$ «Este sentido depende en esta máquina de dos nervios que, sin duda, están compuestos de varios pequeños filamentos [...] pues están destinados a informar al cerebro sobre las diversas acciones de las partes del segundo elemento que [...] darán ocasión para que el alma, cuando estuviere unida a este cuerpo, conciba las diferentes ideas de luces y colores» (AT, XI, 149; Tratado del hombre, 57).

${ }_{17}$ «Pues hay dos cosas en el alma humana de las cuales depende todo el conocimiento que podemos tener de su naturaleza: una es que el alma piensa, la otra que, estando unida al cuerpo, puede obrar y padecer con él. No dije casi nada de esta última y me apliqué solamente a hacer entender bien la primera, porque mi propósito era el de probar la distinción que existe entre el alma y el cuerpo, para lo cual aquella solamente pudo servir y la otra hubiera sido perjudicial» (AT, III, 664; a Elisabeth, 21 de mayo de 1643).
} 
na ${ }^{18}$ - le es propio ver colores, escuchar sonidos, sentir hambre, etc. Este tipo de percepción es factible gracias a la unión psicofísica del sujeto. Sin embargo, en este nivel, la captación de las sensaciones de color, olor, etc., implican cierta traducción, es decir, una interpretación en la que intervienen ambas sustancias y que difiere de las propiedades auténticas de las cosas. Esta intervención protagónica del sujeto le otorga prioridad, pues reinterpreta el mundo natural articulando lo perceptible y lo percibido, lo visual y lo visible.

A partir de la discontinuidad entre el dato meramente fisiológico y la traducción que el sujeto cognoscente debe realizar para interpretar el dato y asumirlo como una percepción, Benítez afirma que las propiedades como el color, el sonido y la textura resultan oscuros, confusos e inadecuados para el conocimiento científico, pues no son atributos esenciales de la res extensa. La razón es que dichas propiedades son sensibles propios, es decir, captados por un sentido, y no aportan un conocimiento sobre el mundo natural tal cual es; mientras que los sensibles comunes sí lo hacen, pues perciben el tamaño, la figura, la velocidad, etc., características propias de la materia (ver 1993; 23-42).

Si concedemos que los sentidos externos no nos brindan a partir de la percepción de los sensibles propios un dato fiable y científico sobre la naturaleza de la materia - argumento que favorecería la crítica de Benítez-, eso no implica la negación

\footnotetext{
${ }^{18}$ No se ahondará aquí en los numerosos problemas que la unión de estas dos sustancias y su interacción arrojan. Basta, por lo pronto, señalar el fundamento metafísico que recurrentemente sostiene la posibilidad de este hecho: «Pienso que cuando Dios otorga una alma racional a esta máquina, como a continuación expondré, otorgará a esta alma como sede principal el cerebro y hará que su naturaleza sea tal que tenga sensaciones diversas, según las distintas formas en que estén abiertas las entradas de los poros situados en la superficie del cerebro» (AT, XI, 143; Tratado del hombre, 50).
} 
de una estructura cognitiva unitaria del pensar. En este sentido, Simmons afirma que, a pesar de las insalvables complicaciones que este planteamiento contrae, el sujeto epistémico cartesiano presume de cierta capacidad para conocer la realidad a través de su experiencia sensorial, según la cual sensación e intelecto construyen conjuntamente (ver 2003; 551). Esto confirma lo que Descartes explica en la segunda parte de las Meditaciones con el análisis sobre los atributos o modos del pensamiento, comúnmente llamado entre los estudiosos de la filosofía moderna el 'dilema mental o físico'19.

\section{Conclusiones}

Una de las grandes aportaciones de Descartes fue reconocer que los fenómenos de la luz y el color no son independientes. El pensador francés estaba seguro de haber reducido la óptica a un caso particular de la ciencia del movimiento. La novedad de esta teoría está en la simplificación, pues dirigió sus esfuerzos a tratar que todos los fenómenos ópticos fueran analizados en términos de interacciones mecánicas. El viaje descrito en la fábula de El Mundo era una consecuencia lógica de sus principios metafísicos fuertemente arraigados, y la Dióptrica, una demostración de la fuerza de su teoría de la luz tanto como de su método.

\footnotetext{
${ }^{19}$ «Qué soy, pues? Una cosa que piensa. ¿Qué es una cosa que piensa? Es una cosa que duda, entiende, concibe, afirma, niega, quiere, no quiere y, también, imagina y siente. Ciertamente, no es poco, si todo eso pertenece a mi naturaleza [...] Pero se me dirá que esas apariencias son falsas y que estoy durmiendo: bien; sea así. Sin embargo, por lo menos es cierto, que me parece que veo la luz, que oigo ruido y que siento calor; esto no puede ser falso, y esto es, propiamente, lo que en mí se llama sentir, y esto, precisamente, es pensar» (AT, IX, 22; Meditaciones, 102).
} 
Por supuesto, las problemáticas que implica su teoría de la luz y el color son variadas. Sin embargo, 'el mundo' cartesiano constituyó un paso decisivo en el intento de alejarse de la visión aristotélica realista de los colores y en aclarar el camino para una explicación mecánica de los mismos. A partir de él, se originó una brecha entre el mundo tal y como lo experimentamos y el mundo descrito por la ciencia; dicho espacio sólo se ha agrandado desde entonces. Como afirma Wolf (ver 1993; 49), es un hecho histórico que todos los que le sucedieron discutieron si los colores deberían entenderse como percepción de la configuración de las superficies de los objetos - en el caso de la propiedad de la luz-como un estado mental solamente en quien percibe, o como un 'poder' en los objetos. Sin embargo, la postura tradicional del color ya no se consideraba.

A pesar de los intentos por lograr una explicación de los colores en el orden de la geometría y las matemáticas, Descartes sólo brindó una explicación paradigmáticamente cualitativa, al carecer de medios para calcular la velocidad angular de las partículas y para determinar las propiedades físicas de cada color dentro del espectro. Esta tarea fue la gran aportación de Isaac Newton, quien sustituyó el tamaño del corpúsculo de luz por la velocidad angular del glóbulo, punto de partida que se arraiga en aquel tronco robusto y pleno de ramas cargadas de frutos.

\section{BIBLIOGRAFÍA}

Fuentes:

Adam, Ch. \& Tannery, P. (1964). Oeuvres de Descartes. Paris: Vrin. 
Traducciones:

Descartes, R. (1986). Discurso del método, dióptrica, meteoros y geometría. Guillermo Quintás (trad.) Madrid: Alfaguara. Descartes, R. (1980). Tratado del hombre. Guillermo Quintás (trad.) Madrid: Editorial Nacional.

Descartes, R. (1986). El mundo o tratado de la luz. Laura Benítez (trad. y est. int.) México: UNAM.

Descartes, R. (1995). Los principios de la filosofía. Guillermo Quintás (trad.) Madrid: Alianza Editorial.

Descartes, R. (1977). Meditaciones metafísicas con objeciones y respuestas. Vidal Peña (trad.) Madrid: Alfaguara.

Bibliografía secundaria:

Benítez, L. (1993). El mundo en René Descartes. México: UNAM. Cerantola, S. (2004). «La ley física de Ibn Sahl: estudio y traducción parcial de su Kitāb al-harraqāt». Anaquel de Estudios Árabes 15; 57-95.

Ribe, N. (1997). «Cartesian Optics and the Mastery of Nature». Isis 1 (88); 42-61.

Sabra, A. I. (1981). Theories of Light, from Descartes to Newton. London: Cambridge University Press.

Shea, W. R. (1993). La magia de los números y el movimiento. La carrera científica de Descartes. Pedro Campos (trad.) Madrid: Alianza Editorial.

Simmons, A. (2003). «Descartes on the Cognitive Structure of Sensory Experience». Philosophy and Phenomenological Research 3 (67); 549-579.

Smith, M. (1987). «Descartes's Theory of Light and Refraction: A Discourse on Method». The American Philosophical Society 3 (77); 1-92.

Stansfield, B. (1984). «Descartes on Refraction: Scientific versus Rhetorical Method». Isis 3 (75); 481-502. 
Straker, S. (1970). «Optics and Methodology in the 17th Century». Science, New Series 3962 (170); 1074-1076.

Westfall, R. S. (1962). «The Development of Newton's Theory of Color». Isis 3 (53); 339-358.

Wolf-Devine, C. (1993). Descartes on Seeing. Epistemology and Visual Perception. Illinois: The Journal of the History of Philosophy. 


\section{RESUMEN}

La fábula propuesta por Descartes en El Mundo o Tratado de la Luz es una hipótesis que intenta explicar diversos fenómenos del mundo natural a partir de los principios del mecanicismo. La primera parte del trabajo contextualiza históricamente el problema y expone los principios básicos del mecanicismo cartesiano. En la segunda sección, se aborda la explicación mecanicista cartesiana sobre la percepción del color, la cual se enmarca en la visión de un universo pleno de materia, constituido por partes que responden a las leyes de la mecánica. En este singular universo, el fenómeno de la luz representa paradigmáticamente la comunicación del movimiento a través de un medio adecuado.

Palabras clave: color; Descartes; Tratado de la Luz; mecanicismo; luz.

\section{ABSTRACT}

Descartes' fable The World or The Treaty of the Light is one hypothesis that tries to explain various phenomena that happen in the natural world, having as a starting point the principles of mechanisism. The first part of this work historically contextualizes the problem and exposes the basic principles of Cartesian mechanisism. In the second section, the Cartesian mechanisist explanation about the perception of color is developed, which is enmarked in a vision of a universe full of matter, constituted by parts that respond to the laws of mechanics. In this singular universe, the phenomenon of the light paradigmatically represents the communication of movement through an adequate media. 
Key words: color; Descartes: Treaty of the Light; mechanisism; light. 